\title{
Paediatric Crossword Puzzle - 22 (Answers)
}

Manouri Senanayake ${ }^{1}$

Sri Lanka Journal of Child Health, 2014; 43(2): 115

\begin{tabular}{|c|c|c|c|c|c|c|c|c|c|c|c|c|}
\hline & & $1 R$ & ${ }^{2} \mathrm{~A}$ & $\mathbf{P}$ & I & ${ }^{3} \mathbf{D}$ & & ${ }^{4} \mathbf{T}$ & & \multicolumn{3}{|c|}{${ }^{5 D}$} \\
\hline & & & $\mathbf{N}$ & & & 0 & & $\mathbf{H}$ & & & 0 & \\
\hline & & & $\bar{A}$ & & & $\mathbf{U}$ & & $\mathbf{R}$ & & & $\mathbf{M}$ & \\
\hline${ }^{6} \mathbf{P}$ & ${ }^{7} \mathbf{A}$ & $\mathbf{U}$ & $\mathbf{L}$ & & & ${ }^{8} \mathbf{B}$ & $S$ & $\mathbf{E}$ & $\mathbf{R}$ & & I & \\
\hline & $S$ & & & & & $\mathbf{L}$ & & $\mathbf{E}$ & & & $\mathbf{N}$ & \\
\hline & C & & & 90 & ${ }^{10} \mathrm{~N}$ & $\mathbf{E}$ & & & ${ }^{11} \mathrm{~S}$ & & $\mathbf{A}$ & \\
\hline${ }^{12} S$ & I & $X$ & . & & $\mathbf{A}$ & . & & ${ }^{13} \mathrm{C}$ & H & I & $\mathbf{N}$ & \\
\hline & $\mathbf{T}$ & & & & 14 I & $\mathbf{J}$ & $15 \mathrm{~V}$ & & A & & $\mathbf{T}$ & \\
\hline${ }^{16} \mathbf{N}$ & I & $\mathbf{N}$ & ${ }^{17} \mathbf{E}$ & & & & $\mathbf{E}$ & & L & & & ${ }^{18} \mathbf{F}$ \\
\hline & C & & $\mathbf{M}$ & & & & $\mathbf{L}$ & & $\mathbf{L}$ & & & $\mathbf{L}$ \\
\hline & & & ${ }^{19} \mathbf{L}$ & $\mathbf{E}$ & $\mathbf{U}$ & ${ }^{20} \mathrm{C}$ & 0 & C & 0 & $\mathbf{R}$ & I & A \\
\hline $21 \mathrm{E}$ & & & A & & & K & & & W & & & $\mathbf{T}$ \\
\hline $\mathbf{E}$ & & & & & & D & & & & & & \\
\hline $22 \mathrm{G}$ & 0 & $\mathbf{0}$ & D & & ${ }^{23} \mathbf{P}$ & $\mathbf{U}$ & $\mathbf{M}$ & $\mathbf{P}$ & $\mathbf{K}$ & I & $\mathbf{N}$ & \\
\hline
\end{tabular}

${ }^{1}$ Senior Professor in Paediatrics, Faculty of Medicine, University of Colombo 